\title{
Design of Golden-section adaptive control for ship course-based mathematical modeling
}

\author{
Hongbing Qin, Peirong Ge \\ Marine college of Shandong jiaotong university, Weihai, Shandong,China
}

Keywords: Ship steering controller Golden; golden-section adaptive control; Simulink tool

\begin{abstract}
The development history of this paper on automatic pilot, domestic and foreign research review and summarize the situation. Analysis of Dynamics equation of ship maneuvering motion based on mathematical models of wind, waves and mathematical model of flow disturbance. Simply introduces the principle of ship course control, while traditional mathematical model and design process of PID autopilot. Design of ship steering controller in Matlab Simulink Toolbox for the simulation, simulation results show that, in the case of controllers parameters do not change still achieved good results.
\end{abstract}

\section{Introduction}

In the 1960 of the 20th century, with classical control theory and electronics of development and the application, appeared has set control technology and electronic devices $\mathrm{Yu}$ one of second generation automatically rudder, that PID automatically rudder, due to PID automatically rudder has control parameter less, structure simple and the itself inherent must of robustness, features, in ship heading control system in the made has very good of control effect, based on these advantages, thereafter 30 years of most large ship are installation has PID automatically rudder.

And yiqian of mechanical type automatically rudder compared PID automatically rudder although has has is big of improve, but it itself also has insufficient of at: a is PID automatically rudder is to precise mathematics model for premise Xia design of, and ship model parameter will with mount, speed, depth, factors of different and changes, outside interference also will often occurred change, so ship of mathematics model and outside interference model exists with many uncertainty.

To determine model design of PID automatically rudder no since adapted capacity, in sailing in the manipulation personnel hard adjustment optimal of PID parameter, because ship of model parameter is with outside changes of, this must requirements manipulation personnel kept of according to sailing State and outside environment for parameter adjustment; II is Dang outside interference exists high frequency waves Shi, usually PID automatically rudder easy caused high frequency turned rudder, will greatly increased rudder machine of wear, increased ship of resistance, should as reduced high frequency turned rudder; Third, when a ship is in very harsh environments, such as heavy sea sailing, due to considerable uncertainty exists, PID autopilot often produce large rudder angle of the rudder, can cause the ship's yaw, this situation is very dangerous, and can even lead to serious accidents.

In view of ship motion model parameter uncertainty. in starting process of adaptive control system, how to ensure the stability of the closed-loop system and dynamic quality, golden-section Adaptive ship course control methods are proposed. It is the golden ratio and minimum variance combined with the feedback control strategy. This method is simple, convenient, robust, smooth transition process, easy to implement.

\section{Control principle of PID autopilot}

An automatic steering control system, from its steady-state and dynamic characteristics of two parts to judge the effects of good or bad. Steady-state characteristics of the system mainly refers to the steady-state error, System Dynamics refers to the stability of the system and the quality of the transition process. When the autopilot to maintain heading direct from the wind, waves, flow 
disturbance, between the actual ship and command the sailing has a certain bias, this is the steady-state errors, autopilot control goal is to try to make the error decreases. In addition, the system also exist such as: drift of the amplifier, shadow of the measuring element, the implementation of components of friction and structures, such as errors, autopilot systems for small-signal when there is a dead zone, a minimum amount of signal is called sensitivity.

When outside interference so that when the ship's yaw less sensitivity, control of rudder angle no response, sensitivity than steady-state error of the autopilot system. Sensitivity at different conditions need to set a different value, sea state is good, smaller set of sensitivity to be able to improve the accuracy of course; when sea conditions are poor, choose a larger sensitivity in order to avoid frequent steering.

In order to save energy, minimize the change of course when sailing, on course to be determined as far as possible; On the, heading control precision also cannot set of too high, due to ship in sailing in the certainly will by wind, waves, flow, interference, if on heading of control precision requirements high, will kept of turned rudder to Amendment heading deviation, for example ship on waves of high frequency components interference too sensitive, on will caused frequently playing rudder phenomenon, such will greatly increased fuel of consumption and increased rudder machine of wear, so in design automatically rudder Shi on need heading stable and energy consumption Zhijian found a best of balance index. After a wild and other academics found that this modest and consume far more energy than the waves by steering inertia resistance caused by environmental interference, such as the consumption of energy, especially ships steer high frequency waves under clear conditions caused by interference such as energy consumption of the inertial resistance is negligible.

Norrbin considers the stability of the ship and steers the frequency proposed a ship's course-keeping steering performance cost function.

Classic of PID control is control field application most widely of control algorithm, it algorithm simple, control parameter meaning clear, itself has must of robustness, advantages get has many experts scholars of favored. This section to with rudder machine institutions of a order linear wild this model for research object, design has PID automatically rudder, used Matlab since with of NCD Toolbox on PID parameter for optimization. in design PID automatically rudder Shi General are is used a order linear wild this model as research object. PID parameters can be obtained through experience, but it is not an optimal method. at present there are several popular PID parameter optimization method, we use Matlab toolbox NCD (Nonlinear Control Design Blockset) method, this method is accurate, simple and avoids the otherwise extensive programming and complex calculations.

\section{Design and establishment of mathematical model for ship motion}

With shipping industry of development, port increasingly busy, on various ship control manipulation of requirements LI benefits improve. and for as marine oil mining, geological exploration, power positioning ship, on heading, track of control precision requirements is more high. this on requirements we established a compared precise of mathematics model. in actual application in the, we will according to different of purpose select different complex degree of model. usually to simplified problem, controller of design Shi usually used simplified of mathematics model, These models can be derived from a complex model, its effectiveness can be ship trials to verify.

Ground is an inertial coordinate system, as shown in Figure 1 in the three dimensional Cartesian coordinate system e core powder almond, e is the origin, and can be an arbitrary point on the Earth's surface, a shaft is to point to the Earth, the two axis in a plane perpendicular to this coordinate system within classical physics can be applied to analyze the system. 


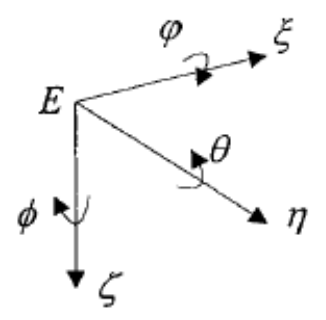

Fig .1 Coordinate system diagram

Hull coordinate system is a moving coordinate system, o for original points, can in hull Office take is as original points, in Figure 2 by shows of hull coordinate system in the, longitudinal axis Ox parallel Yu ship cross shake axis and points to bow, horizontal not parallel Yu longitudinal axis and points to starboard, vertical axis Oz points to ship end of. to research problem convenient, usually put ship gravity g set for hull coordinate system of original points, Ox and Oz respectively for ship of cross shake axis, longitudinal shake axis and first shake axis.

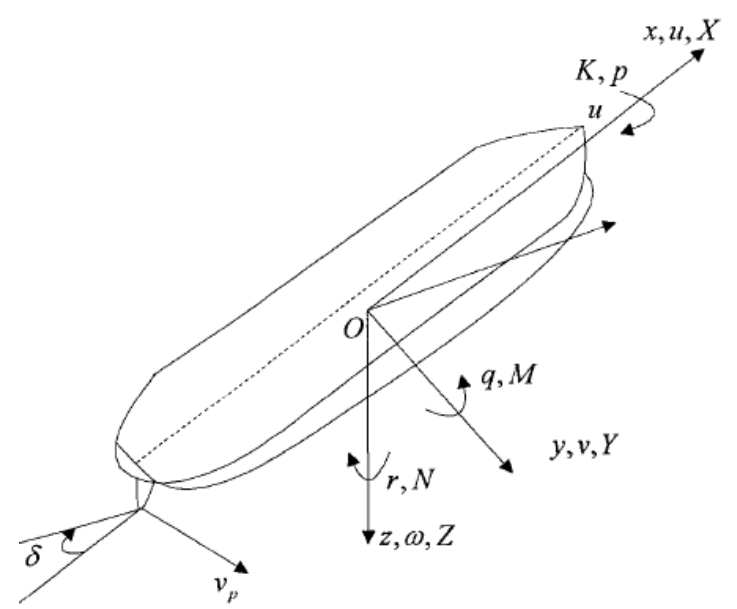

Fig .2 Ship heading control system simulation

We usually can put for for ship of forces into two class. a is water power, another a is non-water power of forces. generally words, role Yu hull of water power and torque will with itself geometry shape, hull movement characteristics also and fluid itself characteristics about. If research in limit channel in the of manipulation sex problem, is also should including Bank, end of interference force. on in wave Shang of manipulation sex problem, is also will involved wave rejection power,. now we only consider on a a agreed of ship type, in given of fluid in the movement of situation .

1957 Japan wild Professor Qian maneuvering motion based on systems of linear equations, from the perspective of control engineering to study the ship's maneuverability problems. manipulation caused by changing the rudder movement, as the output response of maneuvering to enter the rudder angle, according to the mathematical model of Steering response and characterization of ship maneuverability index is presented.

Sea breeze on hull of disturbance except can through waves produced on ship of force and torque, also can directly role Yu water above of hull part, produced on ship of force and torque. by sea breeze produced of effect can independent of into two part to processing, they is stability fan and turbulence wind. in research ship control system in the, stability fan can as constant value disturbance to processing, turbulence wind and random waves characteristics more close, so turbulence wind can with analysis random waves of method to analysis.

Due to changes in speed and direction of ocean currents is very slow, so the role of ocean currents, the ship is relatively stable, in the short term, currents can be handled as a constant disturbance. ship movement systems, ocean currents caused by ships will be an additional disturbance around the $\mathrm{Oz}$ axis,

Waves interference is caused ship swing of most main disturbance, is interference force in the most complex of a, so, many situation Xia consider of outside interference refers to of is waves interference, General put waves interference force is divided into two part, a is a order waves 
interference force, another a is second-order waves interference force. Dang waves of site value not is big, caused ship of swing in can control range within, ship by and waves frequency same and and bogaocheng linear relationship of waves force called a order waves interference force; II order waves thousand rejection force also that waves drift force.

Ship model parameter perturbation wave interference common situations and the outside world, heading for parameter optimization PID control of a major overshoot, rudder control Horn rudder greatly; Golden-section adaptive control on the course is well maintained in the desired direction, and no major overshoot, rudder than larger, but still within a reasonable range, control effect is satisfactory. Simulation results as shown in Figure 3 and Figure 4

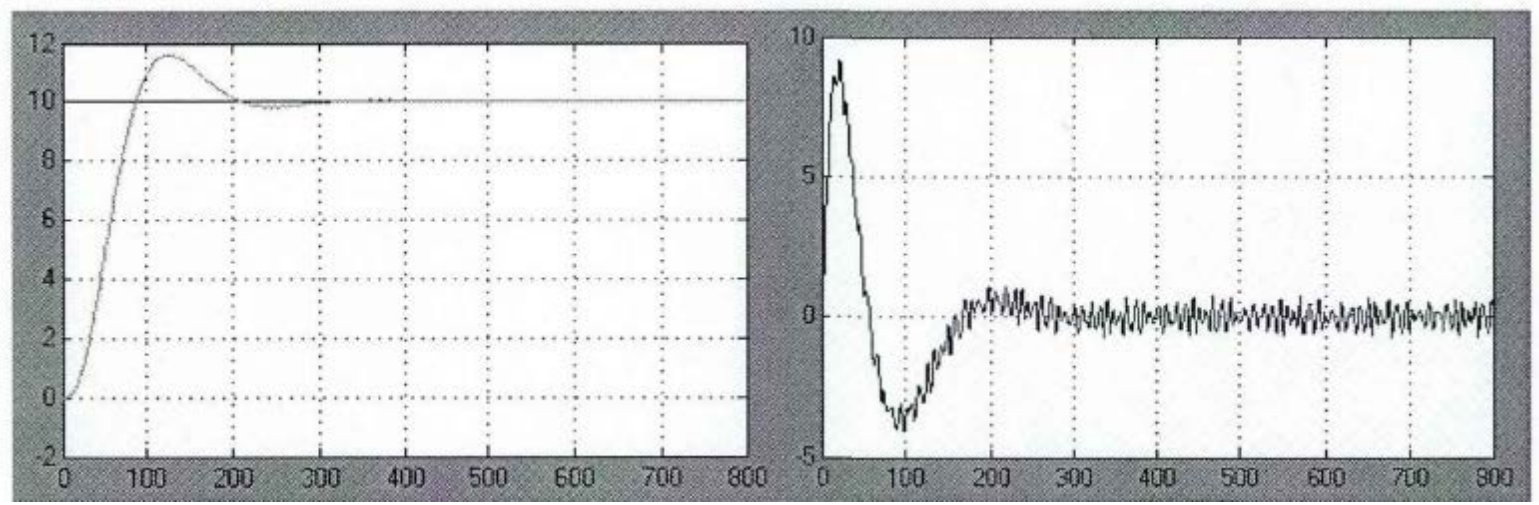

Fig .3 PID control simulation course and rudder angle change curve simulation

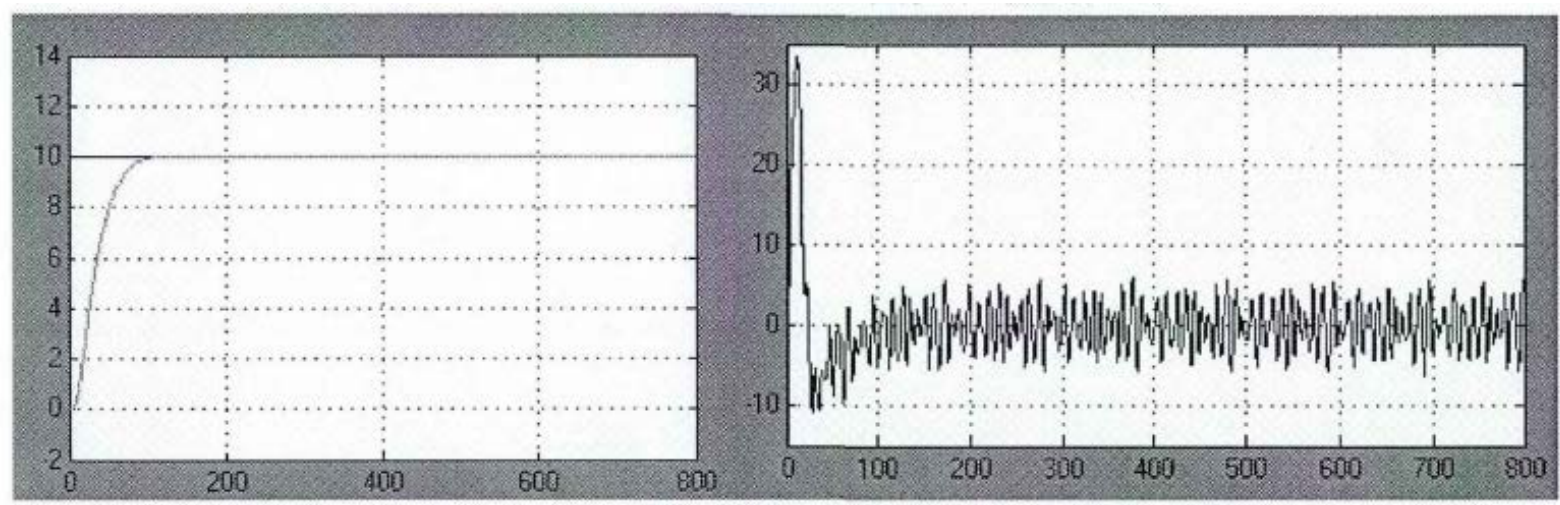

Fig .4 Golden-section adaptive control the course and rudder angle curve

Above, we can obtained such of conclusion, dang ship model parameter is fixed not variable of situation Xia, with NCD optimization parameter PID control effect and gold segmentation since adapted control effect difference is unlikely to, even PID control effect better some, but Dang ship of model parameter appeared big of photo moving and exists outside interference Shi, PID control effect quickly variable poor, and gold segmentation since adapted control effect changes not is big, control precision is high, That by using the golden section method to design controller for uncertain control object has a good adaptability, unknown parameters and unknown interference, control parameters remain unchanged to maintain good control effect and is very robust.

\section{References}

[1]DoK.D., panJ., Jiang Z.-p. Global exponential tracking control of underaetuated surface ships in the body frame. Proceedings of the American Control Conference. Anchorage, Alaska, USA 2012:4702-4707

[2]Xiaohong Jiao. Nonlinear analysis and design of the system. the Publishing House of electronics industry. 2008.8:126-154 
[3]Sun Duoqing, Wu Hongxin. uniform asymptotic stability of four-order time-varying discrete systems. control theory and its applications, 2006,23 (6): 19-26.

[4] Peking University Department of mathematics, geometry and algebra research laboratory group for "Higher Algebra" Higher Education Press

[5] Tongji University Department of Mathematics for Engineering Mathematics -- "linear algebra" fourth edition, higher education press

[6] T.B.Maples and G.A.Spanos.Performance Study of a Selevtive Encryption Scheme for the Security of Networked,Real-time Video. In Proceedings of The 4th International Conference on Computer Communications and Network, September 2008 